\title{
MicroRNA-363-3p inhibits tumor cell proliferation and invasion in oral squamous cell carcinoma cell lines by targeting SSFA2
}

\author{
LIANGMING ZHU ${ }^{1}$, LEI ZHANG ${ }^{2}$, YING TANG $^{3}$, FANG ZHANG $^{1}$, CHAO WAN $^{1}$, LIANG XU $^{1}$ and PING GUO ${ }^{1}$ \\ ${ }^{1}$ Department of Stomatology, Yijishan Hospital, The First Affiliated Hospital of \\ Wannan Medical College; ${ }^{2}$ Jiangcheng Dental Clinic; ${ }^{3}$ Department of Endocrinology, \\ Wuhu Hospital of Traditional Chinese Medicine, Wuhu, Anhui 241000, P.R. China
}

Received January 11, 2020; Accepted June 10, 2020

DOI: $10.3892 /$ etm.2021.9981

\begin{abstract}
The aim of the present study was to evaluate the expression levels of microRNA (miR)-363-3p and its underlying physiological function in oral squamous cell carcinoma (OSCC). miR-363-3p expression levels were measured in OSCC cell lines using reverse transcription-quantitative PCR. The role of miR-363-3p in OSCC cells was examined using gain-of-function assays in vitro. Cell proliferation was assessed using Cell Counting Kit-8, 5-ethynyl-2'-deoxyuridine assays and flow cytometry. Cell migration and invasion were evaluated in wound-healing and Transwell Matrigel assays. In addition, bioinformatics analysis predicted binding sites of miR-363-3p on sperm-specific antigen 2 (SSFA2). Luciferase reporter and RNA pull-down assays were conducted to test whether miR-363-3p interacted with SSFA2. miR-363-3p expression was downregulated in OSCC cell lines compared with that in the normal epithelial cell line (NHOK). Additionally, miR-363-3p overexpression suppressed OSCC cell proliferation, migration and invasion in vitro. SSFA2 was verified as a direct target of miR-363-3p, and SSFA2 overexpression partially counteracted the inhibitory effects of miR-363-3p on cell proliferation, migration and invasion in OSCC cell lines. Thus, miR-363-3p may serve as a tumor suppressor via targeting SSFA2 and may represent a potential therapeutic target for OSCC.
\end{abstract}

Correspondence to: Dr Ping Guo, Department of Stomatology, Yijishan Hospital, The First Affiliated Hospital of Wannan Medical College, 2 Zheshan West Road, Wuhu, Anhui 241000, P.R. China E-mail: yisgp163@163.com

Abbreviations: OSCC, oral squamous cell carcinoma; miR-363-3p, microRNA-363-3p; SSFA2, sperm-specific antigen 2; RT-qPCR, reverse transcription-quantitative PCR; OD, optical density; WT, wild-type; mut, mutant

Key words: oral squamous cell carcinoma, microRNA-363-3p, sperm-specific antigen 2

\section{Introduction}

Oral squamous cell carcinoma (OSCC) is the most common type of oral cancer and a major cause of head and neck squamous cell carcinoma (HNSCC)-related morbidity and mortality worldwide (1-3). Although diagnosis and treatment modalities for OSCC, such as chemoradiotherapy, surgery and photodynamic therapy, have progressed $(4,5)$, the 5-year survival rate of patients with OSCC has not substantially improved over recent decades $(2,6,7)$. In addition, the mechanisms underlying the onset and progression of OSCC are not fully understood. Therefore, further studies are required for the improvement of early diagnosis and targeted therapy of OSCC.

Previous studies have suggested that microRNAs (miRNAs; miRs) influence the onset and progression of tumors, including OSCC, and can often be used as diagnostic and prognostic biomarkers (8-14). For example, Wu et al (15) demonstrated that the miR-375/solute carrier family 7 member 11 axis regulates OSCC cell proliferation and invasion. In addition, Cao et al (16) suggested that miR-375 inhibits OSCC cell migration and invasion by targeting platelet-derived growth factor-A. Among these miRNAs, miR-363-3p has been identified as a tumor suppressor in several types of cancer (17-20). However, whether miR-363-3p also serves a role in OSCC remains unclear.

Sperm-specific antigen 2 (SSFA2), also referred to as $\mathrm{K}$-Ras-induced actin-interacting protein, is upregulated in human colon cancer (21). A previous study has described the critical roles of SSFA 2 as a potential target in human diseases, particularly in cancer (22). Moreover, P38/MAPK inhibition downregulates SSFA2 expression and promote the progression of lymphoma (23).

In the present study, the expression levels and the function of miR-363-3p were evaluated in OSCC cell lines. The findings of the present study suggested that miR-363-3p could inhibit the proliferation, migration and invasion of OSCC cell lines. The potential downstream target and regulatory mechanism of miR-363-3p were also examined. miR-363-3p exerted its effect on OSCC cell lines by negatively regulating SSFA 2 expression levels. Thus, the present study provided insight into the role of miR-363-3p in OSCC. 


\section{Materials and methods}

Cell culture. The human SCC-9 (cat. no. CRL-1629), SCC-25 (cat. no. CRL-1628) and Cal-27 (cat. no. CRL-2095) OSCC cell lines were purchased from American Type Culture Collection. The immortalized normal human oral keratinocyte (NHOK) were a gift from Dr Suyin Hu, Department of Endocrinology, Wuhu Hospital of Traditional Chinese Medicine's (Wuhu, China) laboratory by dissociated from the tissue at Passage 4 (24). Cells were cultured in DMEM (Gibco; Thermo Fisher Scientific, Inc.) supplemented with $10 \%$ fetal bovine serum (Gibco; Thermo Fisher Scientific, Inc.) at $37^{\circ} \mathrm{C}$ in a humidified atmosphere of $5 \% \mathrm{CO}_{2}$.

Cell transfection. The miR-363-3p mimic, pcDNA3.1-SSFA2 overexpression vector, as well as and their negative controls were obtained from Shanghai GenePharma Co., Ltd. SSFA2 overexpression plasmid, empty plasmid vector, mimic NC or miR-363-3p mimic were transfected into SCC-9 and SCC-25 cells using Lipofectamine ${ }^{\circledR} 2000$ (Invitrogen; Thermo Fisher Scientific, Inc.) at final concentration of $10 \mu \mathrm{M}$ according to the manufacturer's protocols. The miR-363-3p mimic with the sense strand sequence 5'-AAUUGCACGGUAUCCAUCUGUA-3' and the mimic NC sense strand sequence 5'-UUGUAC UACACAAAAGUACUG-3' were used for the transient transfection of glioma cells. Reverse transcription-quantitative PCR (RT-qPCR) was performed to assess the transfection efficiency.

$R T$-qPCR. TRIzol ${ }^{\circledR}$ reagent (Invitrogen; Thermo Fisher Scientific, Inc.) was used to extract total RNA from SCC-9 and SCC-25 cells. Equal amounts of RNA were reverse transcribed into cDNA using the BeyoRT ${ }^{\mathrm{TM}}$ II First Strand cDNA Synthesis Kit (Beyotime Institute of Biotechnology) according to the manufacturer's protocols. The PCR program was as follows: $42^{\circ} \mathrm{C}$ for $15 \mathrm{~min}$, followed by $85^{\circ} \mathrm{C}$ for $5 \mathrm{sec}$. The expression levels of miR-363-3p and SSFA2 were then quantified by RT-qPCR, using One Step SYBR PrimeScript RT-PCR kit (cat. no. DRR096A; Takara Bio, Inc.). The thermocycling conditions consisted of an initial denaturation at $95^{\circ} \mathrm{C}$ for $10 \mathrm{~min}$, followed by 40 cycles at $95^{\circ} \mathrm{C}$ for $15 \mathrm{sec}$ and $60^{\circ} \mathrm{C}$ for $30 \mathrm{sec}$. U6 was used as the reference gene for miR-363-3p and GADPH was used as endogenous control for SSFA2. The $2^{-\Delta \Delta \mathrm{Cq}}$ comparative method (25) was used to normalize the gene expression levels. The specific primers are as follows: miR-363-3p forward, 5'-GCCGAGAATTGCACGGTATC-3' and reverse, 5'-CTCAACTGGTGTCGTGGA-3'; SSFA2 forward, 5'-TGGCAAGAAAGGCCCCTGTG-3' and reverse, 5'-GGAGCAGCAGCAGGATCAGG-3'; GAPDH forward, 5'-ACGGATTTGGTCGTATTGGGCG-3' and reverse, 5'-CTC CTGGAAGATGGTGATGG-3' and U6 forward, 5'-CTCGCT TCGGCAGCACA-3' and reverse, 5'-AACGCTTCACGAATT TGCGT-3'.

Western blotting. After total protein (1 mg) extraction using RIPA buffer (Thermo Fisher Scientific, Inc.), protein quantification was determined by the bicinchoninic acid protein assay method. The equivalent amount of total protein $(5 \mu \mathrm{g})$ was segregated on $10 \%$ SDS-PAGE and subsequently transferred to PVDF membranes (EMD Millipore). Thereafter, the membranes were blocked in $5 \%$ non-fat milk at $37^{\circ} \mathrm{C}$ $1 \mathrm{~h}$, followed by incubation with primary antibody at $4^{\circ} \mathrm{C}$ for overnight and incubation with the secondary horseradish peroxidase-conjugated antibodies (cat. nos. 7074 and 7076; 1:2,000; Cell Signaling Technology, Inc.) at room temperature for $1 \mathrm{~h}$. The bands were visualized using enhanced chemiluminescence reagent (cat. no. SW2030, Beijing Solarbio Science \& Technology Co., Ltd.). The signals of target proteins were normalized to that of $\beta$-actin and GAPDH. The relative expression of the proteins was analyzed using Image J v1.8.0 software (National Institutes of Health). The primary antibodies were as follows: Cyclin D1 (1:1,000; cat. no. ab238625), PCNA (1:1,000; cat. no. ab18197), SSFA2 (1:1,000; cat. no. ab195334), Cox-2 (1:1,000; cat. no. ab15191), MMP-2 (1:1,000; cat. no. ab86607), MMP-9 (1:1,000; cat.no. ab58803), $\beta$-actin (1:1,000; cat. no. ab8226). Antibodies were purchased from Abcam.

Cell Counting Kit-8 (CCK-8) and 5-ethynyl-2'-deoxyuridine $(E d U)$ assays. Cell proliferation $\left(1 \times 10^{4}\right)$ was detected by CCK-8 and EdU assays. Cells (1x10 $)$ were cultured in 96-well plates and CCK- 8 assay was performed according to the manufacturer's instructions (Beyotime Institute of Biotechnology). The optical density values were then measured at $450 \mathrm{~nm}$.

Cells $\left(1 \times 10^{4}\right)$ were cultured in six-well plates and cell proliferation was also determined using EdU assays, which were performed using an EdU Apollo DNA in vitro kit (Guangzhou RiboBio Co., Ltd.), according to the manufacturer's protocols. The cells were visualized under a fluorescence microscope (magnification, x200).

Cell cycle analysis. For cell cycle analysis, OSCC cells were collected and trypsinized after transfection. The cells were fixed in $75 \%$ ethanol at room temperature for $20 \mathrm{~min}$, treated with propidium iodide (Sigma-Aldrich; Merck KGaA) at room temperature for $10 \mathrm{~min}$. The samples were then washed three times with PBS, and cell cycle distribution was subsequently analyzed by flow cytometry BD LSRFortessa ${ }^{\mathrm{TM}}$ X-20 (BD Biosciences) using the FlowJo V10.0 software (BD Biosciences).

Cell migration assay. Transfected cells were cultured until 90\% confluence. Before making the wound, the DMEM medium was replaced with fresh culture medium without FBS. The cells were then scratched using $10-\mu 1$ sterile pipette tips and incubated for $48 \mathrm{~h}$ at $37^{\circ} \mathrm{C}$. The images were then captured at the 0 and $48 \mathrm{~h}$ timepoints after wounding under a light microscope at x200 magnification. The cell migratory ability was quantified by measuring the width of the advancing margins of cells in three randomly selected microscopic fields.

Transwell assay. Migration and invasion assays were performed using Transwell chambers assay as described previously (26). The chambers were coated with Matrigel (50 $\mu \mathrm{g}$; BD Biosciences) $37^{\circ} \mathrm{C}$ for $4 \mathrm{~h}$. A total of $\sim 1 \times 10^{6}$ cells were added to the top chamber of 24 -well plates in serum-free medium. The bottom chamber was filled with complete medium supplemented with $20 \%$ FBS. After 24-h incubation, the invasive or migratory cells were fixed with $4 \%$ formaldehyde at room temperature for $15 \mathrm{~min}$ and stained with $0.1 \%$ crystal violet at 

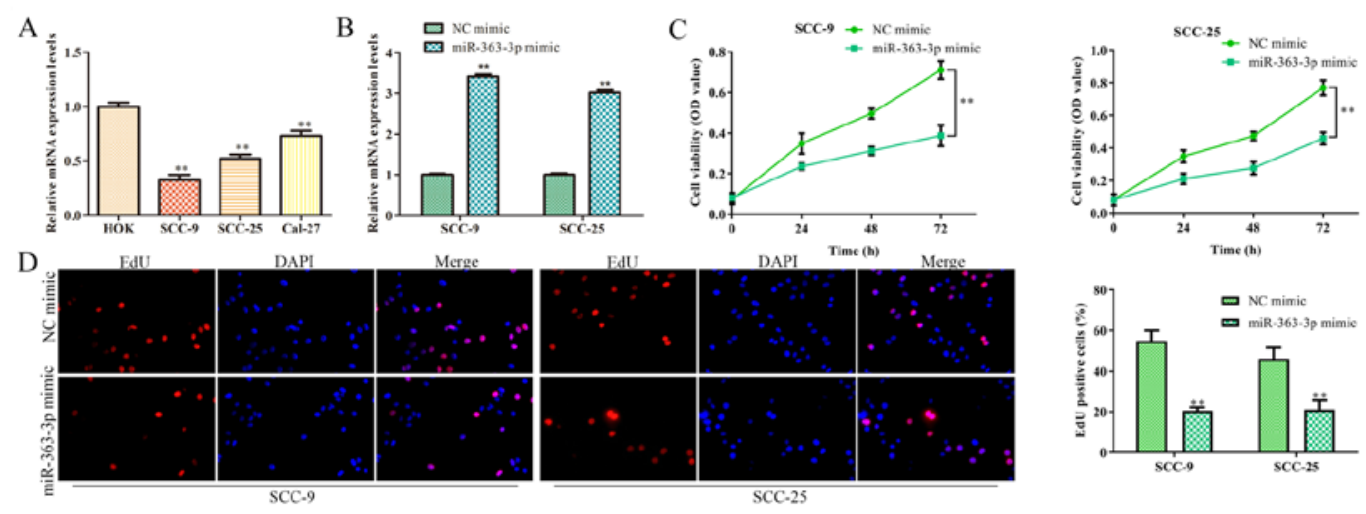

$\mathrm{E}$
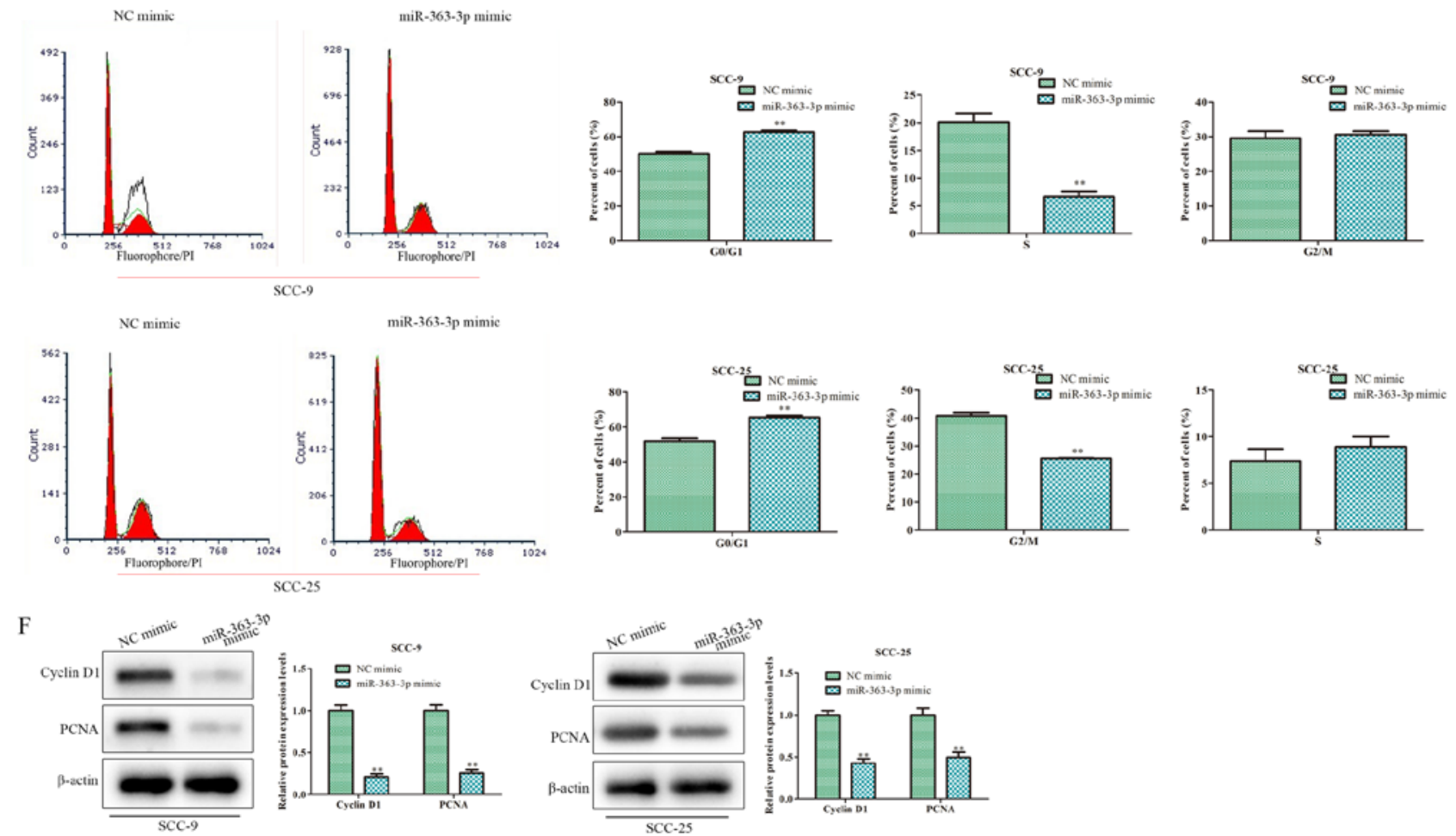

Figure 1. miR-363-3p inhibits the proliferation of OSCC cell lines. (A) Expression levels of miR-363-3p in OSCC cell lines and the HOK normal epithelial cell line. ${ }^{* * *} \mathrm{P}<0.05$ vs. NHOK cells. (B) Reverse transcription-quantitative PCR was performed to assess transfection efficiency. (C) Cell Counting Kit-8 and (D) EdU assays were conducted to examine cell proliferation. Magnification x200. (E) Effects of miR-363-3p on cell cycle progression were examined by flow cytometry. (F) Protein expression levels of cyclin D1 and PCNA were measured in OSCC cell lines by western blotting. Data are presented as the mean \pm SD; ${ }^{* *} \mathrm{P}<0.01$ vs. NC mimic. EdU, 5-ethynyl-2'-deoxyuridine; miR, microRNA; NC, negative control; OD, optical density; OSCC, oral squamous cell carcinoma; PCNA, proliferating cell nuclear antigen.

room temperature for $20 \mathrm{~min}$. Transwell migration assay was performed using a similar protocol but without Matrigel. The cells were then counted and imaged from five fields of view per chamber under a light microscope (magnification, x200). Migration (Invasion) rate $(\%)=$ Migrated $($ Invaded) cells $/$ total cells $\times 100 \%$.

Luciferase reporter assay. Bioinformatics tool Starbase v2.0 (https://starbase.sysu.edu.cn/) was used to predict SSFA 2 binding-sites for miR-363-3p. The 3'untranslated region (UTR) of SSFA2 containing the wildtype (WT) or mutant (Mut) binding sites for miR-363-3p were cloned into pmirGLO reporter vector (Promega Corporation) to make the SSFA2-WT or SSFA2-Mut plasmids, respectively. The SCC-9 and SCC-25 cell lines were co-transfected with $150 \mathrm{ng}$
pmirGLO luciferase reporter vectors (Promega Corporation) containing SSFA2-wild-type (WT) or SSFA2-mutant (Mut) (Shanghai GenePharma Co., Ltd.), together with miR-363-3p mimic or mimic NC at final concentration of $10 \mu \mathrm{M}$ using Lipofectamine ${ }^{\circledR} 2000$ (Invitrogen; Thermo Fisher Scientific, Inc.). The luciferase activities were detected at $48 \mathrm{~h}$ post-transfection by the Dual-luciferase ${ }^{\circledR}$ reporter assay system (Promega Corporation)with Renilla luciferase activities used as internal control.

RNA pull-down assay. Cells were transfected with WT-bio-miR-363-3p, MUT-bio-miR-363-3p or Bio-NC (GE Healthcare Dharmacon, Inc.) labeled with $50 \mathrm{nM}$ biotin using Lipofectamine ${ }^{\circledR} 2000$ (Thermo Fisher Scientific, Inc.), which were obtained using biotin RNA Labeling 

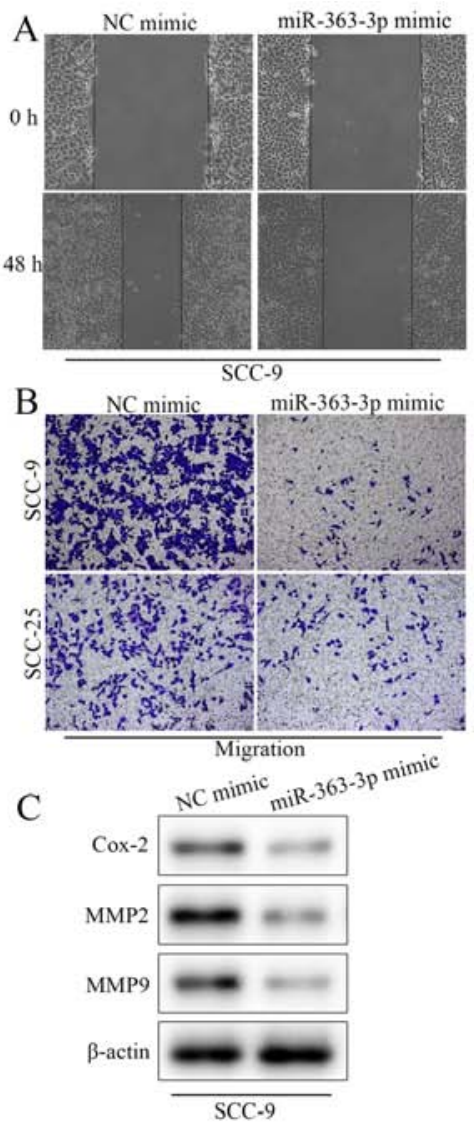
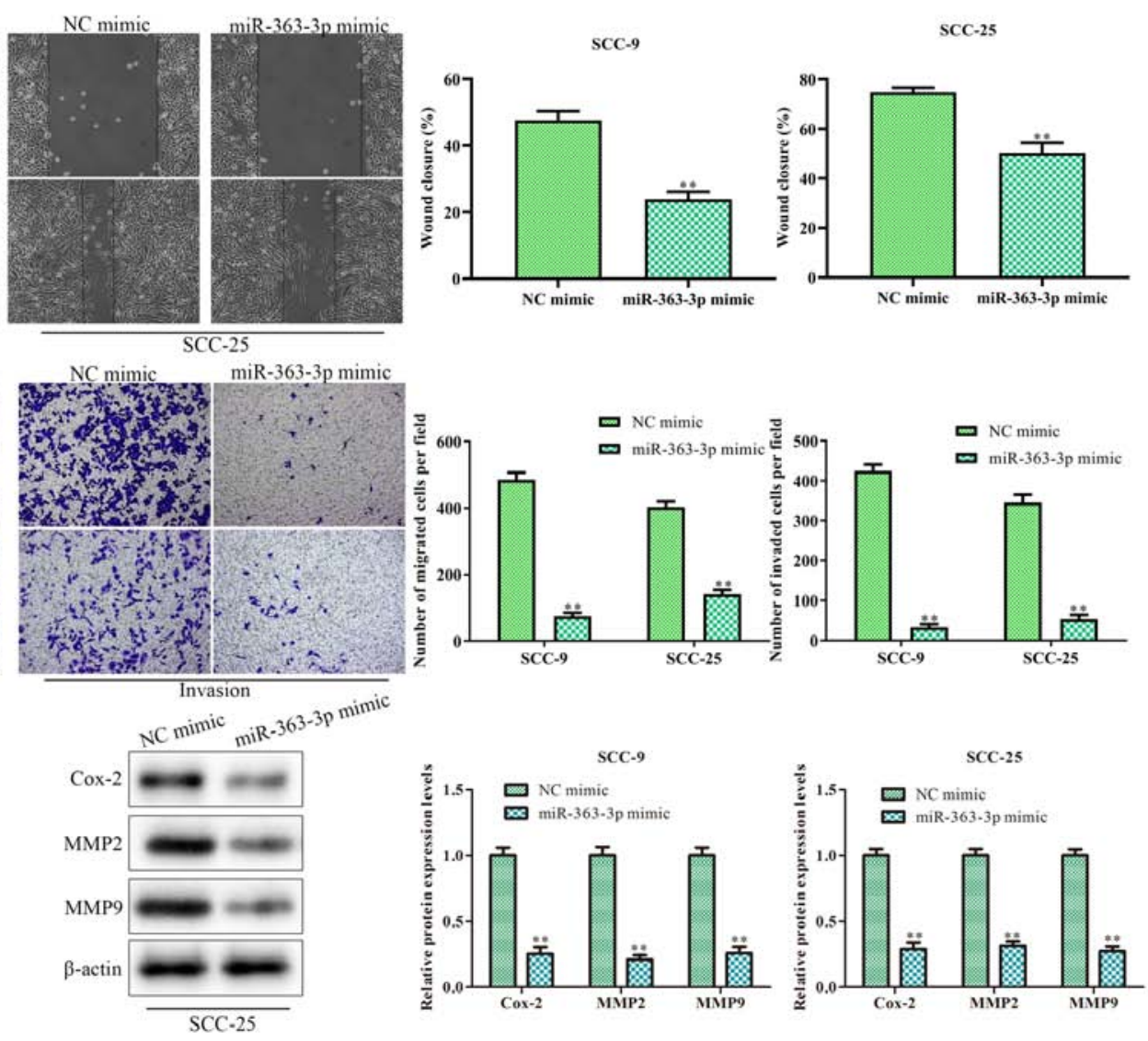

Figure 2. miR-363-3p inhibits OSCC cell migration and invasion. (A) Wound healing assays were carried out to measure cell migration rates. (B) Cell migration and invasion was evaluated using Transwell and Matrigel assays, respectively. (C) Western blotting assays were carried out to assess the protein expression levels of Cox-2, MMP2 and MMP9. Magnification, x200. Data are presented as the mean $\pm \mathrm{SD} ;{ }^{* *} \mathrm{P}<0.01 \mathrm{vs}$. NC mimic. Cox-2, cyclo-oxygenase-2; miR, microRNA; MMP, matrix metallopeptidase; NC, negative control; OSCC, oral squamous cell carcinoma.
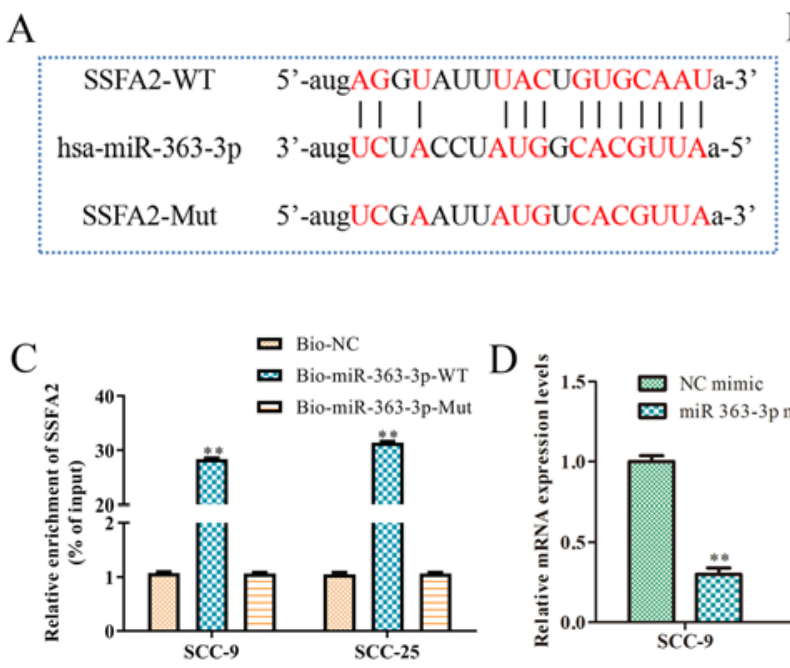
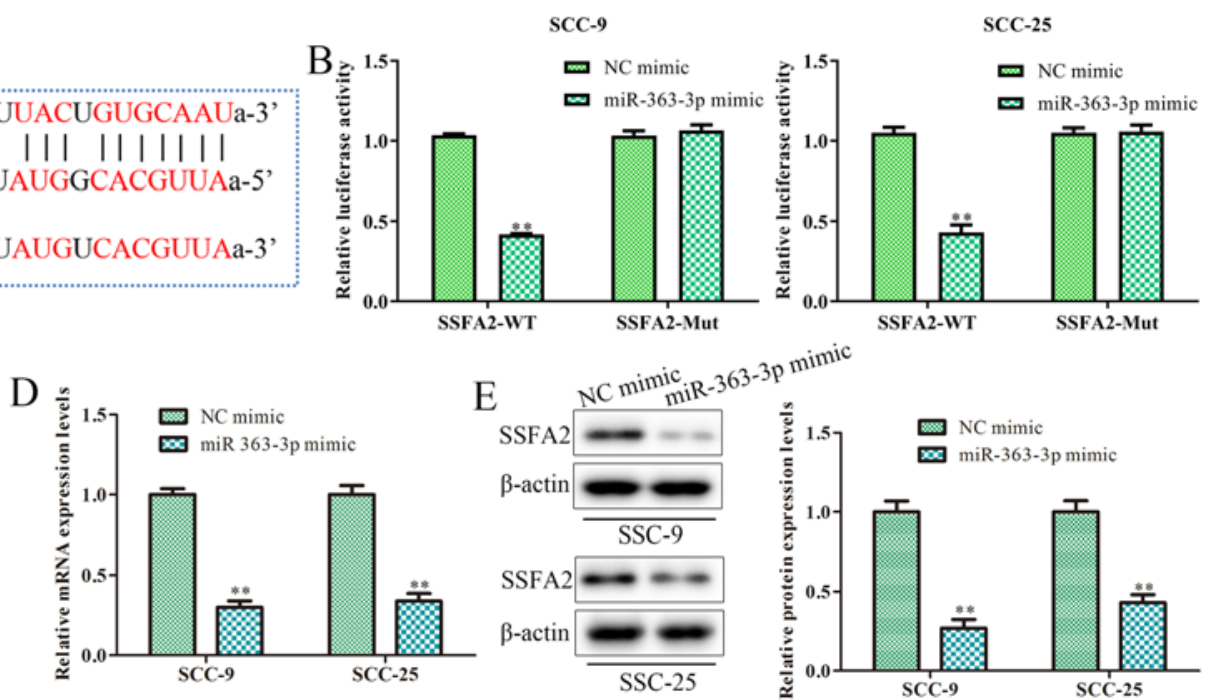

Figure 3. miR-363-3p targets SSFA2. (A) Predicted target sites of miR-363-3p in the SSFA2 3' untranslated region. (B) Luciferase reporter assay was conducted to verify the relationship between miR-363-3p and SSFA2. (C) RNA pull-down assay was carried out to confirm the interaction between miR-363-3p and SSFA2. ${ }^{* *} \mathrm{P}<0.01$ vs. Bio-NC. (D) Reverse transcription-quantitative PCR and (E) western blotting analyses of SSFA2 expression levels in OSCC cell lines following transfection with the miR-363-3p mimic. Data are presented as the mean $\pm \mathrm{SD} ;{ }^{* *} \mathrm{P}<0.01 \mathrm{vs}$. NC mimic. miR, microRNA; mut, mutant; NC, negative control; OSCC, oral squamous cell carcinoma; SSFA2, sperm-specific antigen 2; WT, wild-type.

Mix (Roche Diagnostics). Cells $\left(1 \times 10^{4}\right)$ were then pelleted at $1,200 \mathrm{x} \mathrm{g}$ for $5 \mathrm{~min}$ at room temperature after transfected for $48 \mathrm{~h}$. The cells were then resuspended in lysis buffer $(20 \mathrm{mM}$ Tris, $5 \mathrm{mM} \mathrm{MgCl}, 100 \mathrm{mM} \mathrm{KCl}, 0.3 \%$ NP-40 and $50 \mathrm{U}$ RNase OUT; Invitrogen; Thermo Fisher Scientific, Inc.) after washing twice with PBS and complete 
A

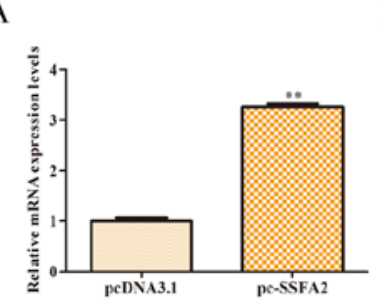

B

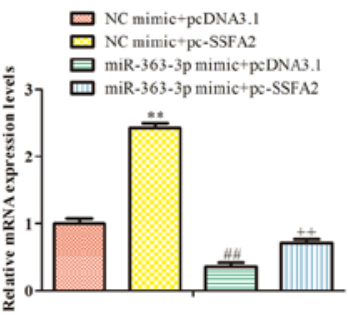

C

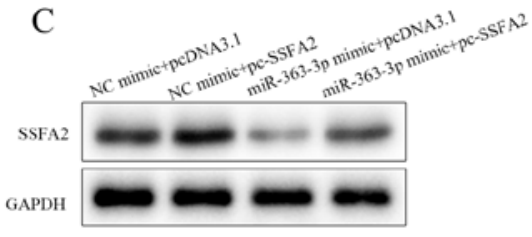

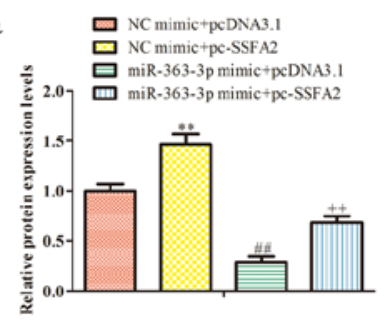

D

E
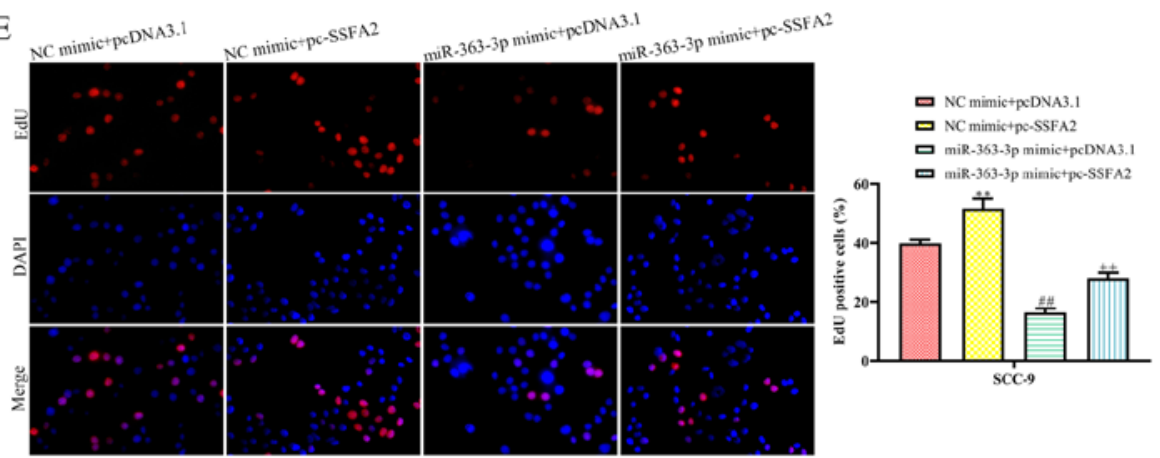

F

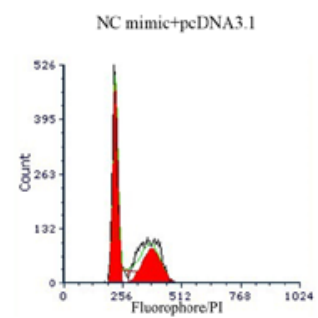

NC mimictpe-SSFA2

miR-363-3p mimic +pcDNA3.1

miR-363-3p mimic + pc-SSFA2
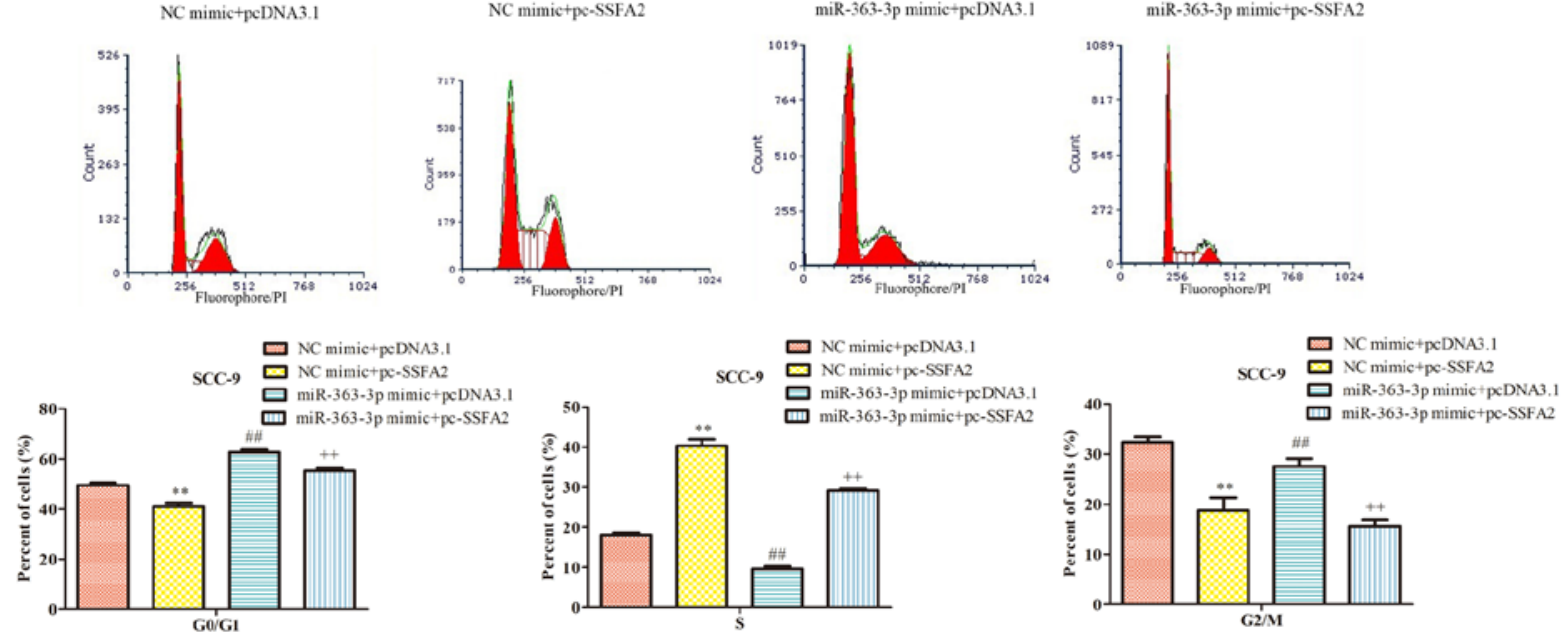

Figure 4. Effect of miR-363-3p in OSCC cell proliferation is mediated by SSFA2. (A) Reverse transcription-quantitative PCR analysis of SSFA2 expression levels in SCC-9 cells following transfection with the pcDNA3.1 or pc-SSFA2. ${ }^{* *} \mathrm{P}<0.05$ vs. pcDNA3.1. (B) Reverse transcription-quantitative PCR analysis of SSFA2 expression levels in SCC-9 cells following transfection with the miR-363-3p mimic or NC mimic and SSFA2 overexpression vector or pcDNA3.1. (C) Western blotting assay was applied to detect the SSFA2 expression level. (D) Cell Counting Kit-8 and (E) EdU assays were performed to evaluate cell proliferation. Magnification, x200. (F) Effects of miR-363-3p and SSFA2 on cell cycle progression were examined using flow cytometry. Data are presented as the mean $\pm \mathrm{SD} ;{ }^{* *} \mathrm{P}<0.01$ vs. $\mathrm{NC}$ mimic + pcDNA3.1; ${ }^{\# \#} \mathrm{P}<0.01$ vs. $\mathrm{NC}$ mimic + pc-SSFA2. ${ }^{++} \mathrm{P}<0.01$ vs. miR-363-3p mimic + pcDNA3.1. EdU, 5-ethynyl-2'-deoxyuridine; miR, microRNA; NC, negative control; OD, optical density; OSCC, oral squamous cell carcinoma; SSFA2, sperm-specific antigen 2.

mini-protease inhibitor cocktail (Roche Applied Science), which was incubated for $5 \mathrm{~min}$ on ice. The cell lysate was isolated and collected by centrifugation at $12,000 \mathrm{x} \mathrm{g}$ for $5 \mathrm{~min}$ at room temperature. Moreover, the miRNA biotin pull-down experiments were conducted according to the previous reports (27). RT-qPCR was used to determine the mRNA levels in the precipitates.

Statistical analysis. Data are presented as the mean \pm SD. All experiments were independently conducted three times and statistical analysis was performed using SPSS 21.0 software (IBM Corp.). Student's t-test was used to determine the significant differences between two groups and one-way ANOVA followed by Tukey's post hoc test were used to analyze differences between $>$ two groups. $\mathrm{P}<0.05$ was considered to indicate a statistically significant difference.

\section{Results}

miR-363-3p inhibits the proliferation of OSCC cells. RT-qPCR was performed to evaluate the expression levels of miR-363-3p in the human SCC-9, SCC-25 and Cal-27 OSCC cell lines, and in the HOK normal epithelial cell line. The expression levels of miR-363-3p were significantly lower in OSCC cell lines, compared with HOK cells (Fig. 1A). As SCC-9 and SCC-25 cells exhibited the lowest miR-363-3p expression levels, these cell lines were used in subsequent experiments.

Additional experiments were conducted to determine the potential effect of miR-363-3p on the proliferation of OSCC cells. miR-363-3p mimic transfection was used to increase the expression level of miR-363-3p, and transfection efficiency was confirmed by RT-qPCR (Fig. 1B). CCK-8 and 

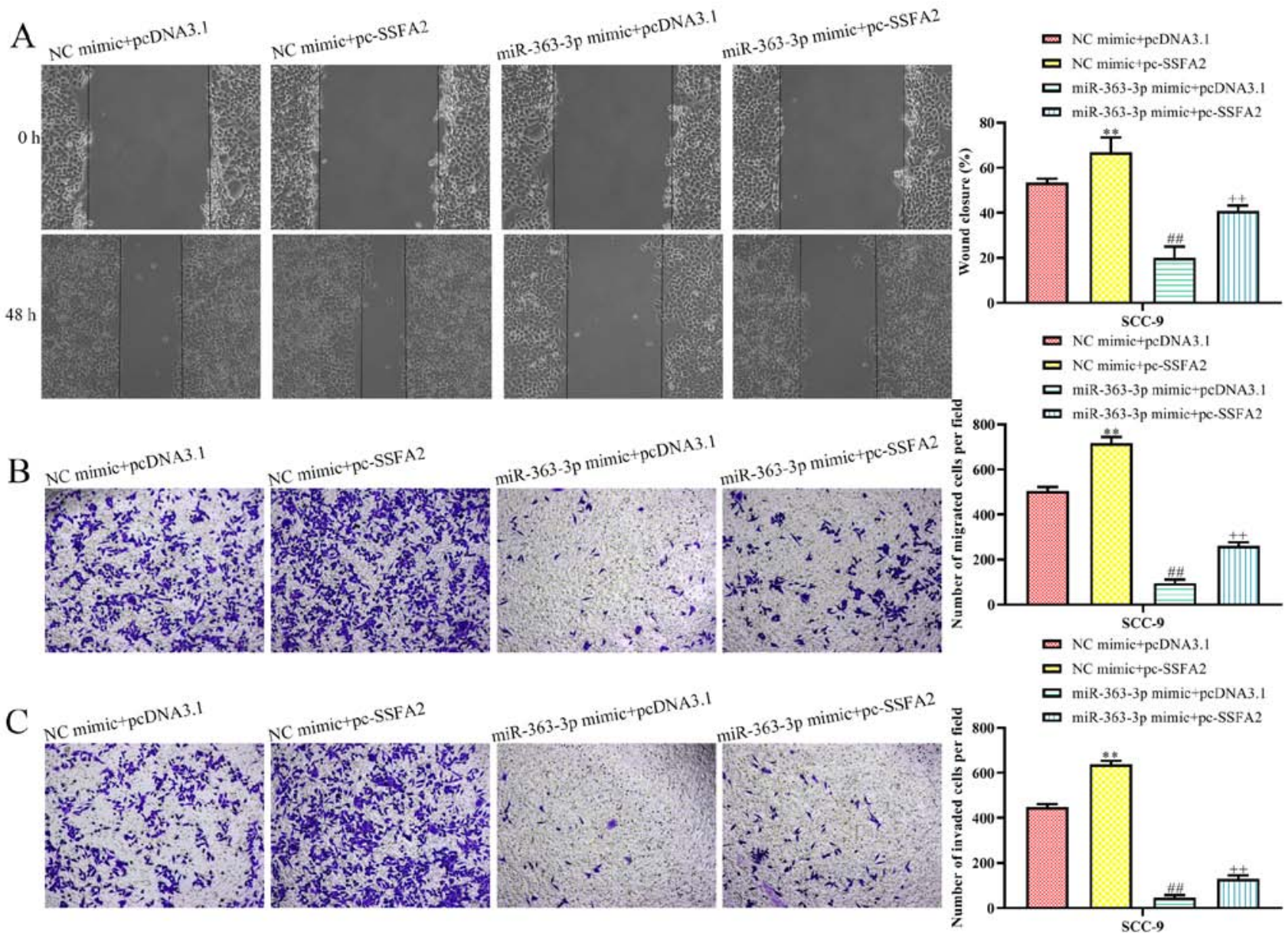

NC mimictpc-SSFA2

口 miR-363-3p mimic-pcDNA3,I $\square$ miR-363-3p mimictpc-SSFA2

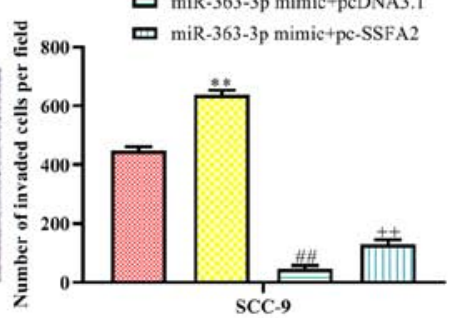

Figure 5. miR-363-3p inhibits OSCC cell migration and invasion by targeting SSFA2. (A) Wound healing assay were performed to determine the migration potential of SCC-9 cells following transfection. (B) Transwell Matrigel assays were applied to explore the migratory ability of OSCC cells. (C) Transwell Matrigel assay were performed to measure the invasive potential of SCC-9 cells following transfection. Magnification, $\mathrm{x} 200$. Data are presented as the mean $\pm \mathrm{SD} ;{ }^{* *} \mathrm{P}<0.01$ vs. $\mathrm{NC}$ mimic + pcDNA3.1; ${ }^{\# \#} \mathrm{P}<0.01$ vs. $\mathrm{NC}$ mimic + pc-SSFA2. ${ }^{++} \mathrm{P}<0.01$ vs. miR-363-3p mimic + pcDNA3.1. miR, microRNA; NC, negative control; OSCC, oral squamous cell carcinoma; SSFA2, sperm-specific antigen 2.

EdU assays demonstrated that cell proliferation was significantly reduced following transfection with the miR-363-3p mimic, compared with the NC mimic-transfected groups (Fig. 1C and D, respectively). In addition, the number of cells in $\mathrm{G}_{0} / \mathrm{G}_{1}$ phase significantly increased, whilst the proportion of SCC-9 cells at the S phase was significantly decreased (Fig. 1E). No significant differences were observed at the $G_{2}$ phase following miR-363-3p overexpression. The proportion of SCC-25 cells at the $G_{2}$ phase was significantly decreased but no significant differences at the S phase when miR-363-3p was overexpressed (Fig. 1E). Moreover, the expression levels of proliferation markers cyclin D1 and PCNA were decreased in the presence of miR-363-3p mimic, compared with NC mimic (Fig. 1F). Altogether, these results suggested that miR-363-3p overexpression suppresses the proliferation of OSCC cells.

miR-363-3p inhibits OSCC cell migration and invasion. The role of miR-363-3p in OSCC cell migration and invasion was assessed using wound healing and Transwell Matrigel assays, as well as western blotting. miR-363-3p overexpression significantly decreased cell migration in the OSCC cell lines, compared with the respective NC mimic-transfected group (Fig. 2A and B). Additionally, miR-363-3p mimic transfection inhibited the invasive abilities of SCC-9 and SCC-25 cells (Fig. 2B). Furthermore, western blotting was performed to detect the expression levels of Cox-2, MMP-2 and MMP-9, proteins implicated in invasion (17). miR-363-3p overexpression resulted in decreased levels of Cox-2, MMP-2 and MMP-9 in SCC-9 and SCC-25 cell lines compared with expression levels in the respective control groups (Fig. 2C). These results suggested that miR-363-3p may inhibit the migration and invasion potential of OSCC cells.

SSFA2 is a target of miR-363-3p. To better understand the mechanism underlying the effect of miR-363-3p on OSCC cells, Starbase 2.0 was used, where it was determined that SSFA2 was a potential target of miR-363-3p (Fig. 3A). To confirm this potential interaction between SSFA2 and miR-363-3p, a luciferase reporter assay and an RNA pull-down experiment were performed. Transfection with the miR-363-3p mimic reduced the activity of SSFA2-WT by $>50 \%$, but not the activity of the SSFA2-Mut reporter (Fig. 3B). Moreover, the RNA pull-down assay indicated that SSFA2 was only pulled by bio-miR-363-3p-WT (Fig. 3C). Consistent with 
the aforementioned results, RT-qPCR and western blotting also suggested that miR-363-3p overexpression significantly decreased the expression of SSFA2 in SCC-9 and SCC-25 cells, both at the mRNA and the protein levels (Fig. 3D and E, respectively). Collectively, these findings indicated that SSFA2 is a target of miR-363-3p.

Effect of miR-363-3p on OSCC cell proliferation by targeting SSFA2. To determine whether the functional role of miR-363-3p in SSC-9 cells was mediated by SSFA2, co-transfection experiments were conducted using the miR-363-3p mimic and an SSFA2 overexpression vector; SSFA2 overexpression was confirmed by RT-qPCR (Fig. 4A). Transfection with miR-363-3p mimic and empty pcDNA3.1 vector significantly decreased SSFA 2 mRNA and protein expression levels compared with the control group (Fig. 4B and C). By contrast, SSFA2 overexpression partially reversed the effect of miR-363-3p on these expression levels. Moreover, the CCK-8 assay indicated that cell proliferation was significantly reduced following transfection with the mimic and empty vector (Fig. 4D); however, proliferation was restored following co-transfection with pc-SSFA2. Data from the EdU assay confirmed these results (Fig. 4E). Moreover, SSFA2 overexpression partially counteracted the inhibitory effects of miR-363-3p on cell cycle progression (Fig. 4F). Thus, it was demonstrated that miR-363-3p may inhibit cell proliferation in OSCC cells lines through SSFA2.

miR-363-3p inhibits the migratory and invasive abilities of OSCC cells via targeting SSFA2. The role of the miR-363-3p/SSFA2 axis in cell migration and invasion was also examined using co-transfection experiments. Results from the wound healing assay demonstrated that the wound closure rate significantly increased following SSFA2 overexpression (Fig. 5A). Transfection with miR-363-3p mimic and empty pcDNA3.1 vector significantly inhibited cell migration; however, the overexpression of SSFA 2 reversed the effects of miR-363-3p on cell migration (Fig. 5A). Similar results were observed in a Transwell migration assay (Fig. 5B). In addition, cell invasion was also significantly decreased by miR-363-3p mimic and partially restored following overexpression of SSFA2 (Fig. 5C). Altogether, these findings suggested that miR-363-3p may inhibit OSCC cell migration and invasion by targeting SSFA2.

\section{Discussion}

In the present study, miR-363-3p expression was significantly downregulated in OSCC cells, compared with a normal epithelial cell line. miR-363-3p overexpression in OSCC cells significantly inhibited cell proliferation, migration and invasion. Additionally, SSFA2 was demonstrated to be a direct target of miR-363-3p, and overexpression of SSFA2 partially reversed the inhibitory effect of miR-363-3p on OSCC cells. These findings suggested that miR-363-3p exerted its inhibitory function in OSCC cells by targeting SSFA2.

Previous studies have described the involvement of miRNAs in OSCC pathogenesis. For example, miR-101 inhibits OSCC growth and metastasis by targeting zinc finger E-box binding homeobox 1 . miR-155 regulates proliferation, cell cycle progression and apoptosis through p27Kip1 in the Tca8113 OSCC cell line (28). Rastogi et al (29) demonstrated that knockdown of miR-377 promoted OSCC growth and migration by targeting histone deacetylase 9 . Downregulated miR-363-3p expression has previously been reported in colorectal cancer (18), head and neck cancer (27), and laryngeal cancer (28), and may function as a tumor suppressor in these malignancies (29). However, to the best of our knowledge, the role of miR-363-3p in OSCC has not yet been studied. In the present study, the expression levels of miR-363-3p were assessed in OSCC cell lines, and gain-of-function assays indicated that overexpression of miR-363-3p reduced OSCC cell proliferation, migration and invasion, suggesting that miR-363-3p may serve as a tumor suppressor in OSCC.

Previous studies have demonstrated that miRNAs can modulate gene expression levels by specifically binding to the 3' untranslated regions of their target mRNAs, thereby repressing gene expression at post-transcriptional level (30). Using bioinformatics analysis, it was demonstrated in the present study that SSFA2 had potential miR-363-3p target sites. SSFA2 has been described as a potential target in colon cancer (21). A recent study has suggested that SSFA2 deletion inhibits cell proliferation and promotes cell apoptosis in glioma (31). Using co-transfection experiments, the present study demonstrated that SSFA2 overexpression partly abrogated the inhibitory effect of miR-363-3p mimic on malignant OSCC. However, in vivo experiments are required to confirm the present findings.

In conclusion, miR-363-3p serves an inhibitory role on the proliferation and invasion of OSCC cells by suppressing SSFA2 expression. Moreover, the discovery miR-363-3p/SSFA2 axis may provide a new diagnostic and therapeutic strategy for OSCC.

\section{Acknowledgements}

The authors would like to express gratitude to Dr Suyin $\mathrm{Hu}$, Department of Endocrinology, Wuhu Hospital of Traditional Chinese Medicine (Wuhu, China), who provided the NHOK cell line.

\section{Funding}

No funding was received.

\section{Availability of data and materials}

The datasets used and/or analyzed during the current study are available from the corresponding author on reasonable request.

\section{Authors' contributions}

PG designed the experiments. LZha, YT, FZ, CW, LZhu and LX performed the experiments and analyzed the data. All authors read and approved the final manuscript.

\section{Ethics approval and consent to participate}

Not applicable. 


\section{Patient consent for publication}

Not applicable.

\section{Competing interests}

The authors declare that they have no competing interests.

\section{References}

1. Shi W, Yang J, Li S, Shan X, Liu X, Hua H, Zhao C, Feng Z, Cai Z, Zhang L and Zhou D: Potential involvement of miR-375 in the premalignant progression of oral squamous cell carcinoma mediated via transcription factor KLF5. Oncotarget 6: 40172-401785, 2015.

2. Zini A, Czerninski R and Sgan-Cohen HD: Oral cancer over four decades: Epidemiology, trends, histology, and survival by anatomical sites. J Oral Pathol Med 39: 299-305, 2010.

3. Wang Q, Gao P, Wang X and Duan Y: The early diagnosis and monitoring of squamous cell carcinoma via saliva metabolomics. Sci Rep 4: 6802, 2014.

4. Omura K: Current status of oral cancer treatment strategies: Surgical treatments for oral squamous cell carcinoma. Int J Clin Oncol 19: 423-430, 2014.

5. D'Souza G and Robbins HA: Sexual and relationship health among survivors of oropharyngeal or oral cavity squamous cell carcinoma. Cancer 123: 1092-1094, 2017.

6. Forastiere A, Koch W, Trotti A and Sidransky D: Head and neck cancer. N Engl J Med 345: 1890-1900, 2001.

7. Jadhav KB and Gupta N: Clinicopathological prognostic implicators of oral squamous cell carcinoma: Need to understand and revise. N Am J Med Sci 5: 671-679, 2013.

8. Zhao S, Yan L, Zhao Z and Rong F: Up-regulation of miR-203 inhibits the growth of cervical cancer cells by inducing cell cycle arrest and apoptosis. Eur J Gynaecological Oncol 40: 791-795, 2019.

9. Karamitopoulou E, Haemmig S, Baumgartner U, Schlup C, Wartenberg $M$ and Vassella E: MicroRNA dysregulation in the tumor microenvironment influences the phenotype of pancreatic cancer. Mod Pathol 30: 1116-1125, 2017.

10. Paliouras AR, Monteverde T and Garofalo M: Oncogene-induced regulation of microRNA expression: Implications for cancer initiation, progression and therapy. Cancer Lett 421: 152-160, 2018.

11. Ganci F, Sacconi A, Manciocco V, Covello R, Benevolo M, Rollo F, Strano S, Valsoni S, Bicciato S, Spriano G, et al: Altered peritumoral microRNA expression predicts head and neck cancer patients with a high risk of recurrence. Mod Pathol 30: 1387-1401, 2017.

12. Katz B, Trope CG, Reich R and Davidson B: MicroRNAs in ovarian cancer. Hum Pathol 46: 1245-1256, 2015.

13. Szabó Z, Szegedi K, Gombos K, Mahua C, Flasko T, Harda K and Halmos G: Expression of miRNA-21 and miRNA-221 in clear cell renal cell carcinoma (ccRCC) and their possible role in the development of ccRCC. Urol Oncol 34: 533.e21-533.e27, 2016.

14. Zhao J, Dong X, Liu QC and Lu Q: Expression of plasma miR-106a in epithelial ovarian cancer and its diagnostic and prognostic significance. Eur J Gynaecol Oncol 39: 769-772, 2018.

15. Wu Y, Sun X, Song B, Qiu X and Zhao J: miR-375/SLC7A11 axis regulates oral squamous cell carcinoma proliferation and invasion. Cancer Med 6: 1686-1697, 2017.

16. Cao ZH, Cheng JL, Zhang Y, Bo CX and Li YL: MicroRNA-375 inhibits oral squamous cell carcinoma cell migration and invasion by targeting platelet-derived growth factor-A. Mol Med Rep 15: 922-928, 2017.
17. Dong J, Geng J and Tan W: miR-363-3p suppresses tumor growth and metastasis of colorectal cancer via targeting SphK2. Biomed Pharmacother 105: 922-931, 2018.

18. Wang Y, Chen T, Huang H, Jiang Y, Yang L, Lin Z, He H, Liu T, Wu B, Chen J, et al: miR-363-3p inhibits tumor growth by targeting PCNA in lung adenocarcinoma. Oncotarget 8 : 20133-20144, 2017.

19. Hu F, Min J, Cao X, Liu L, Ge Z, Hu J and Li X: miR-363-3p inhibits the epithelial-to-mesenchymal transition and suppresses metastasis in colorectal cancer by targeting Sox4. Biochem Biophys Res Commun 474: 35-42, 2016.

20. Chang J, Gao F, Chu H, Lou L, Wang H and Chen Y: miR-363-3p inhibits migration, invasion, and epithelial-mesenchymal transition by targeting NEDD9 and SOX4 in non-small-cell lung cancer. J Cell Physiol 235: 1808-1820, 2020.

21. Inokuchi J, Komiya M, Baba I, Naito S, Sasazuki T and Shirasawa S: Deregulated expression of KRAP, a novel gene encoding actin-interacting protein, in human colon cancer cells. J Hum Genet 49: 46-52, 2004.

22. Kakiuchi S, Daigo Y, Tsunoda T, Yano S, Sone S and Nakamura Y: Genome-wide analysis of organ-preferential metastasis of human small cell lung cancer in mice. Mol Canaer Res 1: 485-499, 2003.

23. Lin Z, Crockett DK, Jenson SD, Lim MS and Elenitoba-Johnson KSJ: Quantitative proteomic and transcriptional analysis of the response to the p38 mitogen-activated protein kinase inhibitor SB203580 in transformed follicular lymphoma cells. Mol Cell Proteomics 3: 820-833, 2004.

24. Min BM, Woo KM, Lee G and Park NH: Terminal differentiation of normal human oral keratinocytes is associated with enhanced cellular TGF-beta and phospholipase C-gamma 1 levels and apoptotic cell death. Exp Cell Res 249: 377-385, 1999.

25. Livak KJ and Schmittgen TD: Analysis of relative gene expression data using real-time quantitative PCR and the 2(-Delta Delta C(T)) method. Methods 25: 402-408, 2001.

26. Li L, Zhu X, Shou T, Yang L, Cheng X, Wang J, Deng L and Zheng Y: MicroRNA-28 promotes cell proliferation and invasion in gastric cancer via the PTEN/PI3K/AKT signalling pathway. Mol Med Rep 17: 4003-4010, 2018.

27. Lal A, Thomas MP, Altschuler G, Navarro F, O'Day E, Li XL, Concepcion C, Han YC, Thiery J, Rajani DK, et al: Capture of microRNA-bound mRNAs identifies the tumor suppressor miR-34a as a regulator of growth factor signaling. PLoS Genet 7: e1002363, 2011.

28. Fu S, Chen HH, Cheng P, Zhang CB and Wu Y: miR-155 regulates oral squamous cell carcinoma Tca8113 cell proliferation, cycle, and apoptosis via regulating p27Kip1. Eur Rev Med Pharmacol Sci 21: 937-944, 2017.

29. Rastogi B, Kumar A, Raut SK, Panda NK, Rattan V, Joshi N and Khullar M: Downregulation of miR-377 promotes oral squamous cell carcinoma growth and migration by targeting HDAC9. Cancer Invest 35: 152-162, 2017.

30. Lu YC, Cheng AJ, Lee LY, You GR, Li YL, Chen HY and Chang JT: miR-520b as a novel molecular target for suppressing stemness phenotype of head-neck cancer by inhibiting CD44. Sci Rep 7: 2042, 2017.

31. Zhu A, Li X, Wu H, Miao Z, Yuan F, Zhang F, Wang B and Zhou Y: Molecular mechanism of SSFA2 deletion inhibiting cell proliferation and promoting cell apoptosis in glioma. Pathol Res Pract 215: 600-606, 2019. 